\title{
Kernos
}

Revue internationale et pluridisciplinaire de religion grecque antique

3| 1990

Varia

\section{Le Daphnéphorion de Phlya, la daphnéphorie béotienne et l'oracle de Delphes}

Ioannis Loucas

\section{(2) OpenEdition}

Journals

\section{Édition électronique}

URL : http://journals.openedition.org/kernos/990

DOI : $10.4000 /$ kernos.990

ISSN : 2034-7871

\section{Éditeur}

Centre international d'étude de la religion grecque antique

\section{Édition imprimée}

Date de publication : 1 janvier 1990

ISSN : 0776-3824

\section{Référence électronique}

Ioannis Loucas, «Le Daphnéphorion de Phlya, la daphnéphorie béotienne et l'oracle de Delphes », Kernos [En ligne], 3 | 1990, mis en ligne le 19 avril 2011, consulté le 30 avril 2019. URL : http:// journals.openedition.org/kernos/990 ; DOI : 10.4000/kernos.990 


\section{LE DAPHNÉPHORION DE PHLYA, LA DAPHNÉPHORIE BÉOTIENNE ET L'ORACLE DE DELPHES}

Dans sa communication au 1er colloque international du C.E.R.G.A. à Athènes en 1987, mon ancien professeur et ami Guy Donnay a souligné l'importance de l'étude monographique des sanctuaires, comme il a appelé cette approche des faits religieux, aboutissant à la "réalité vécue des cultes antiques", "pour la distinguer à la fois des grandes synthèses et des monographies thématiques sur tel dieu, tel héros ou tel type de culte» ${ }^{1}$. Terminant en présentant l'intérêt de ce type d'approche, Guy Donnay concluait de la sorte : «L'étude monographique nous met enfin en garde contre la tentation de considérer les faits religieux attestés par les textes et les monuments comme devant nécessairement s'insérer dans une organisation structurée de croyances et de pratiques, la religion grecque. Au contraire, elle souligne la diversité des particularismes locaux et les aspects divers qu'une même divinité ou des divinités portant le même nom ? - peut prendre selon le lieu où elle est honorée. Dans cette perspective, la question que se pose Madeleine Jost interpelle notre réflexion :

Et si la religion grecque elle-même, au lieu d'être un ensemble bien organisé dont il faut saisir la cohérence, n'était qu'un agrégat de pratiques accumulées au cours des temps ?" 2

Cependant, l'étude monographique d'un lieu de culte, que l'on peut comparer à une observation faite sous une lunette grossissante, comprend parfois ce risque d'amener à parler trop rapidement de particularisme local ou de ne pas saisir exactement en quoi il consiste. Un retour à une vue plus large est indispensable et met souvent en lumière la présence ailleurs de certains acteurs dont on ne supposait qu'une action isolée, topique, ou négligeable, et qui méritent dès lors d'être eux seuls l'objet d'un examen particulier.

L'histoire d'un culte divin peut-elle se faire sans celle des hommes? C'est guidé par ma propre expérience que je réponds négativement à cette question; car pour comprendre le culte de Rhéa-Cybèle à Phlya (ce dème

1 G. DonNaY, L'étude monographique des sanctuaires grecs et la réalité vécue des cultes antiques, in Kernos, 1 (1988), p. 121-127, surtout p. 121.

2 G. DoNNAY, art. cit., p. 126-127 et M. JosT, Sanctuaires et cultes d'Arcadie, Paris, 1985 (Études péloponnésiennes, 9), p. 6. 
rustique au nord-est d'Athènes qui correspond à l'actuelle région de Chalandri), j'ai dû suivre les traces qu'ont pu laisser partout ailleurs en Grèce où ils s'étaient manifestés, les membres d'un génos hiératique de riches propriétaires fonciers, celui des Lykomides ${ }^{3}$.

Aujourd'hui, je tâcherai de montrer la relation des Lykomides avec le culte oraculaire d'Apollon (que je n'avais évidemment pas pu développer dans le cadre de mon étude sur Rhéa-Cybèle), ce qui, je crois, soulignera la nécessité d'entreprendre, à la lumière des documents de nature diverse dont la recherche dispose, des études monographiques des génè ${ }^{4}$.

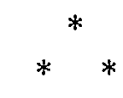

Les Lykomides entrent officiellement dans l'histoire de la Grèce à l'occasion des guerres médiques lorsque, d'après Plutarque ${ }^{5}$ qui cite Simonide, Thémistocle, membre de ce puissant génos d'Attique, fit reconstruire le koเvòv $\tau \hat{\omega} v \Lambda v \kappa o \mu \imath \delta \hat{\omega} v$ télestèrion de Déméter à Phlya qu'avaient incendié les Perses, tandis que le triérarque de la flotte athénienne Lykomédès, fils d'Aischraios, préférait un culte apollinien de Phlya pour manifester sa reconnaissance envers les dieux.

Au dire d'Hérodote ${ }^{6}$ et de Plutarque ${ }^{7}$, Lykomédès fut le premier chef militaire grec à s'être rendu maître d'un vaisseau perse lors de la bataille navale d'Artémision ou de Salamine ${ }^{8}$. Plutarque précise que le triérarque s'était emparé des emblèmes du navire ennemi et les avait consacrés à Apollon Porte-Laurier de Phlya9. Selon Théophraste et Hiéronymos de Rhodes, un élève d'Aristote, cités par Athénée ${ }^{10}$, Apollon y avait un temple où le jeune Euripide, originaire de Phlya, avait exercé

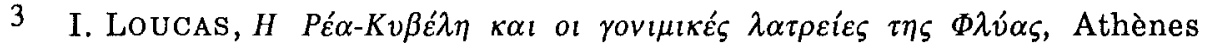
(Chalandri), 1988 (cité ci-dessous Rhéa-Cybèle).

4 Pour les génè d'Attique, pour lesquels il n'y a pas encore de monographies, si ce n'est les articles de la $R E$, on ne dispose que de l'ouvrage de I. TOEPFFER, Attische Genealogie, Berlin, 1889.

5 PLuT., Thémistocle, 1, 4. Cf. aussi infra, n. 9.

6 HDT., VIII, 11.

7 Plut., Thém., 15, 3.

8 Cf. aussi J.K. DAvIEs, Athenian Propertied Families. 600-300 B.C., Oxford, 1971, p. 346, n 9238; LOUCAS, Rhéa-Cybèle, p. 58-59; TOEPFFER, op. cit., p. 209.

9 Cf. aussi W.R. ConNoR, Lycomedes against Themistocles? A Note on Intragenos Rivalry, in Historia, 21 (1972), p. 569-574.

10 ATHÉNÉE, Banquet des Sophistes, X, 424e-f. 
la fonction d'œnochoos. On apprend encore chez Athénée que ce temple consacré à Apollon Délien s'appelait Daphnéphorion et s'ornait de représentations - ou de descriptions - de la fête des Thargélies.

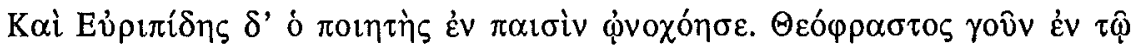

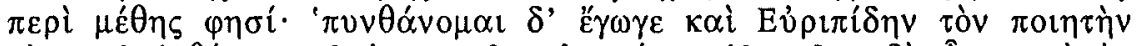

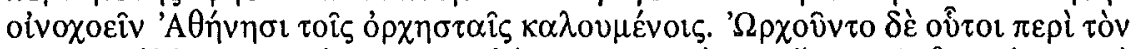

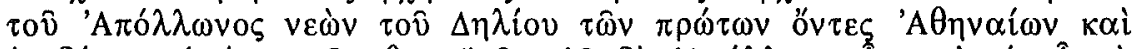

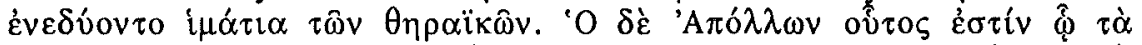

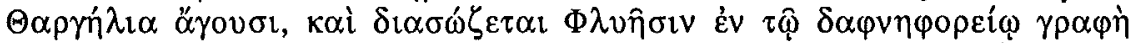

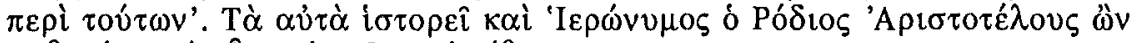

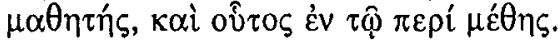

Si l'épithète de «délien» sous laquelle Apollon était vénéré à Phlya nous éloigne du domaine delphique, nous y sommes ramenés par cette mention omniprésente du laurier, qui rappelle tout à la fois le temple mythique d'Apollon à Delphes et la procédure de consultation oraculaire, mais encore les inscriptions commémoratives de victoire aux concours des Thargélies, dédiées à Apollon Pythien ${ }^{11}$. Ces inscriptions ont été découvertes à l'Olympieion, où Pausanias avait vu une statue d'Apollon Pythien ${ }^{12}$.

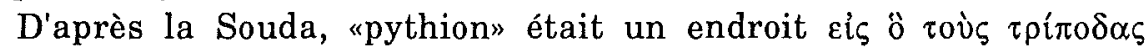

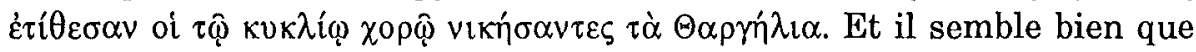
dans le passage d'Athénée rapporté ci-dessus une allusion soit faite à ces danses, puisqu'Euripide avait été l'œnochoos des orchestai qui évoluaient, dansant, autour du Daphnéphorion d'Apollon à Phlya ${ }^{13}$.

On compte aujourd'hui de nombreuses études sur la signification du laurier, qui constituait le fondement du septerion à Delphes, et certainement aussi celui de la daphnéphorie de Phlya, qui est très étroitement associée aux Thargélies ${ }^{14}$. Ces études ont en outre montré la relation existant entre le septerion de Delphes et la daphnéphorie béotienne, qui aboutissait au temple d'Apollon Isménios à Thèbes.

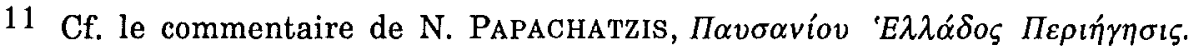

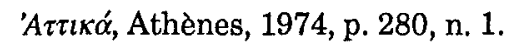

12 Paus., I, 19, 1. Cf. THuc., VI, 55.

13 Cf. LOUCAS, Rhéa-Cybèle, p. 68-69, p. 85.

14 On trouvera des références bibliographiques abondantes dans l'article de C. BÉRARD, Architecture érétrienne et mythologie delphique. Le Daphnéphoreion, in $A K, 14$ (1971), p. 59-73. 
Au dire d'auteurs comme Pindare ${ }^{15}$, Philochore ${ }^{16}$ et Pausanias ${ }^{17}$, le sanctuaire d'Apollon Isménios était situé au sommet de la colline Ismenion, et il y fonctionnait un oracle du dieu qui était l'un des plus réputés de Grèce ${ }^{18}$. Et d'autres témoignages laissent entendre que ce culte thébain d'Apollon n'était pas étranger au génos phlyéen des Lykomides.

Si l'on se tourne d'abord vers Phlya, on apprend par Pausanias 19 que les Nymphes y étaient honorées avec la rare appellation d'Isménides, que ne justifie pas l'historique de ce dème attique ${ }^{20}$. Ce nom, typiquement thébain, s'opposait à celui de divinités considérées à Delphes et ailleurs dans le monde grec comme dispensatrices du pouvoir divin de mantikè mania ${ }^{21}$, pouvoir attribué dans les temps les plus reculés à la Terre Mère ${ }^{22}$. Or cette dernière était particulièrement honorée à Phlya; elle y était tantôt vénérée à côté de Déméter ou de Cybèle, tantôt assimilée à elles deux, en la figure d'une toute-puissante Rhéa - Déméter - Cybèle ${ }^{23}$.

Mais encore, il a dû exister un lien entre les cultes de Phlya, placés sous la tutelle des Lykomides, et ceux de Thèbes, si l'on se réfêre aux activités du Lykomide Méthapos ${ }^{24}$, tenu pour réformateur des mystères

15 PIND., Pyth., IX, 10.

16 PHILOCHORE, apud Schol. SOPH., Ed. Roi, 21.

17 PAUS., IX, 10, 4.

18 Cf. aussi M.P. NiLsson, Griechische Feste, Leipzig, 1906 [Milan, 1975], p. 164165.

19 PAUS., I, 31, 4.

20 Cf. LoucAs, Rhéa-Cybèle, p. 69.

21 Cf., par ex., PAus., X, 12, 11 (à propos du devin béotien Bakis).

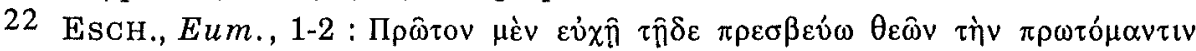
$\Gamma \alpha \hat{i} \alpha v$. Sur Gaia Protomantis, cf. G. Roux, Delphes, son oracle et ses dieux, Paris, 1976, p. 19-34.

23 Cf. LoucAs, Rhéa-Cybèle, passim, ainsi que l'article écrit en commun avec É. Loucas-DURIE, Un autel de Rhéa-Cybèle et la Grande Déesse de Phlya, in Latomus, 45 (1986), p. 392-404 (cité ci-dessous Autel).

24 Sur Méthapos, cf., entre autres, R. BoEHME, Orpheus der Sänger und seine Zeit, Berne-Munich, 1970, p. 314; F.G. Frazer, Pausanias' Description of Greece, Londres, 1898, III, p. 406-407; H.B. HAwES, A Gift of Themistocles : The "Ludovisi Throne» and the Boston Relief, in AJA, 26 (1972), p. 278-306, surtout p. 287-288; LOUCAS, Rhéa-Cybèle, p. 67-81; E. MEYER, art. Phlya, in RE Suppl. X (1965), col. 537; TOEPFFER, op. cit., p. 209, n. 2. 
d'Andanie en Messénie ${ }^{25}$ célébrés en l'honneur d'Apollon Karneios et des Grands Déesses, et de ceux de la ville béotienne ${ }^{26}$. Certains, comme J. G. Frazer ${ }^{27}$, situent les activités de Méthapos au IVe s. av. J.-C., c'està-dire à l'époque de l'hégémonie thébaine; mais ainsi que je l'ai défendu ailleurs ${ }^{28}$, il me paraît plus plausible que le Lykomide en question ait vécu à l'époque archaïque, et plus précisément à l'époque de la seconde guerre de Messénie. Étant donné la réputation de réorganisateur des mystères thébains qu'avait le Lykomide Méthapos, certains savants, comme $\mathrm{H}$. B. Hawes ${ }^{29}$, ont cru pouvoir soutenir l'hypothèse que la «maison de Lykos» à Thèbes, que Pausanias ${ }^{30}$ a vue en ruines à proximité des Portes Proïtides, appartenait non pas au héros béotien Lykos, mais à son homonyme athénien, l'ancêtre - fondateur mythique du génos des Lykomides.

Ce Lykos, fils du roi mythique Pandion, était lui aussi considéré comme un des réorganisateurs des mystères d'Andanie ${ }^{31}$. Et ceux-ci avaient été fondés, disait-on, par son ancêtre Kaukon, fils de Phlyos ${ }^{32}$, Phlyos en qui les Athéniens reconnaissaient le fils de la Terre ${ }^{33}$, et les gens de Phlya, leur revó $\rho \chi \eta \varsigma$ légendaire. Mais c'est bien après les personnages mythiques de Kaukon et Lykos, que les sources situent l'activité de Méthapos ${ }^{34}$.

Et même si la «maison de Lykos» à Thèbes appartenait au héros béotien et non à son homonyme phlyéen (lequel passait aussi pour fondateur du quartier athénien Lykeion où était honoré Apollon Lykeios, d'une certaine manière associé lui aussi à la mantique), le fils

25 PAus., IV, 1, 7. Sur les mystères et les cultes d'Andanie, cf., entre autres,

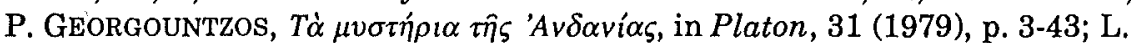
ZIEHEN, Zu den Mysterien von Andania, in Hermes, 60 (1925), p. 338-347.

26 PAUS., IV, $1,7$.

27 Frazer, op. cit., III, p. 406-407. Il est suivi, entre autres, par N. PAPAChatzis,

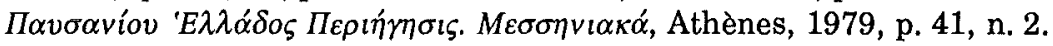

28 LouCAs, Rhéa-Cybèle, p. 78. A. BRUECKNER (Das Reich des Pallas, in MDAI(A),

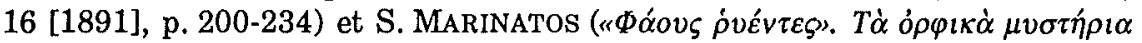
$\pi \eta \hat{\zeta} \Phi \lambda v \alpha_{\alpha}$, in Platon, 2 [1951], p. 228-242, surtout p. 242) situent l'activité de Méthapos à l'époque de Pisistrate.

29 HAWES, art. cit., p. 293.

30 PAUS., IX, 16, 7.

31 PAUS., IV, $1,6$.

32 PAUS., IV, 1,5

33 PAUS., I, 19, 3. Cf. HDT., I, 173.

34 Cf., par ex., L.R. FARnELL, The Cults of the Greek States, Oxford, 1907, IV, p. 117, et les sources p. 358 . 
de Pandion jouissait en propre du renom de devin fameux. C'est bien de cela que Pausanias ${ }^{35}$ se fait l'écho dans ses Phokika, quand il rapporte la tradition selon laquelle se signalent, parmi les chresmologues, le chypriote Euklès, les Athéniens Mousaios, compagnon d'Orphée, et Lykos, fils de Pandion, et le Béotien Bakis ${ }^{36}$, que les Nymphes ont frappé de mania. De son propre aveu, Pausanias a lu les chresmoi de tous ces personnages, si ce n'est ceux de Lykos.

Aux témoignages mentionnés jusqu'ici qui attestent la présence légendaire et/ou historique des Lykomides dans maints cultes apolliniens en divers endroits du monde grec, il faut ajouter un autre élément, à savoir la parenté par alliance du génos sacerdotal de Phlya et celui des Kérykès ${ }^{37}$. Ceux-ci étaient principalement connus pour les fonctions qui leur revenaient dans les mystères d'Éleusis, dans le cadre officiel desquels se sont introduits les Lykomides à partir de l'époque héllénistique, époque à laquelle la charge éleusinienne de dadouque était exercée par les descendants de Thémistocle dispersés dans divers dèmes d'Attique ${ }^{38}$.

En ce qui concerne les cultes apolliniens attiques et delphique, les Kérykès détenaient depuis l'époque de Dracon une charge sacerdotale au temple d'Apollon Délien ${ }^{39}$, et l'on se rappellera que ce même dieu était vénéré au Daphnéphorion de Phlya, où il y avait des descriptions des Thargélies. Or des inscriptions découvertes dans le sanctuaire delphique associent encore les Kérykès à l'Apollon Pythien ${ }^{40}$, qui se rapporte lui aussi aux Thargélies. C'étaient également les Kérykès qui prenaient la tête du cortège partant d'Attique pour le sanctuaire de Delphes ${ }^{41}$.

Si la parenté des Lykomides avec les Kérykès n'est pas antérieure à l'époque hellénistique, les relations entre ces deux génè sacerdotaux d'Attique doivent remonter plus haut dans le temps, puisque, comme

35 PAUS., $\mathrm{X}, 12,11$.

36 Cf. aussi supra, n. 21.

37 Sur les Kérykès, cf., entre autres, K. Clinton, The Sacred Officials of the Eleusinian Mysteries, Philadelphie, 1974 (TAPhS, 64, 3), p. 9, 66-67, 76-86; G. MYlONAS, Eleusis and the Eleusinian Mysteries, Princeton, 1961 (1974), p. 232-235; TOEPFFER, op. cit., p. 86-88.

38 Paus., I, 37, 1. Cf. Clinton, op. cit., p. 50-58; Davies, op. cit., p. 219; LouCAS, Rhéa-Cybèle, p. 87-88; ID., Ritual Surprise and Terror in Ancient Greek Possession-Dromena, in Kernos, 2 (1989), p. 97-104, surtout, p. 97.

39 Cf. P. FouCART, Les mystères d'Éleusis, Paris, 1914, p. 157 et n. 2.

40 Ibid., p. 157.

41 Ibid., p. 157 et n. 3. 
l'ont montré P. Foucart ${ }^{42}$ et K. Clinton ${ }^{43}$, les Kérykès étaient eux aussi un ancien génos attique.

Les cultes de Phlya, que régissaient les Lykomides, avaient un caractère essentiellement populaire et ils s'inscrivent tous dans un contexte commun, à savoir le domaine de la mantique inspirée, celle des poètes mystiques et des devins individuels. Nos sources affirment clairement que les Lykomides étaient avant tout des prêtres renommés de l'orphisme ${ }^{44}$, courant religieux auquel sont rattachés des personnages qui se sont distingués par leur pouvoir oraculaire. Ainsi en va-til du légendaire Mousaios, chresmologue de grand renom, cité par Pausanias à côté du fils de Pandion Lykos ${ }^{45}$. Quant aux personnages historiques, il me suffira de mentionner le contemporain de Pisistrate Onomacrite, que les tyrans athéniens, fondateurs d'un sanctuaire d'Apollon Pythien près du temple de Zeus Olympien ${ }^{46}$, chargèrent de la rédaction des poèmes d'Orphée et des oracles de Mousaios. Lui-même devin renommé, Onomacrite a, chez Plutarque ${ }^{47}$, son nom lié à «des poètes qui entouraient le sanctuaire de Delphes et (qui) recueillaient les réponses, s'en emparaient et tressaient sur le champ vers et cadences autour d'elles pour les y enfermer comme dans des vases» 48 .

Or, à l'époque de Pisistrate, les prêtres orphiques Lykomides avaient joué un rôle actif dans la stabilisation du tyran au pouvoir, ainsi que dans l'organisation de sa propagande politique et religieuse ${ }^{49}$. Et

42 Ibid., p. 156-158.

43 CLINTON, op. cit., p. 115-116.

44 Paus., I, 22, 7; IV, 1, 5; IX, 27, 2; IX, 30, 12. Cf. aussi HipPolyte, Contre les Hérésies, V, 20, qui affirme que les cérémonies de Phlya étaient imprégnées de l'enseignement orphique de Mousaios et de Linos; cf. LouCAS, Autel, passim; ID., Rhéa-Cybele, p. 169-186 et passim.

45 Cf. supra, n. 21 et 35.

46 Selon THUc., VI, 55, Pisistrate, le fils d'Hippias, avait consacré à Apollon Pythien un autel dont certaines parties, découvertes en 1877 à $200 \mathrm{~m}$. au sud du

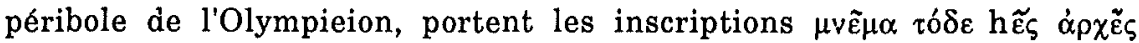

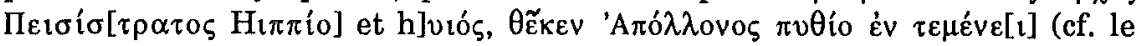
commentaire de Pausanias par PAPACHATZIS, op. cit. (Attika), p. 280, n. 1). C'est dans cette même région qu'ont été également découvertes les bases des monuments chorégiques des Thargélies dont il est question plus haut (cf. n. 11).

47 Plut., Pythie, 25 (407b-c).

48 Cf. aussi M. DELCOURT, L'oracle de Delphes, Paris, 1955 [1981], p. 94.

49 Cf. notamment BoEHME, op. cit., p. 314-317. À consulter également, l'étude de A. ShapIRo, Oracles-Mongers in Peisistratid Athens, ici-même. 
certains savants, comme R. Böhme $\mathrm{e}^{50}$, vont même jusqu'à soutenir qu'Onomacrite est à compter parmi ces Lykomides du VIe s. av. J.-C.

Les sources que j'ai été amené à exploiter pour étayer ces quelques remarques m'ont fait parler de l'Apollon Daphnéphoros de Phlya. Mais les Phlyéens vénéraient encore le dieu sous le nom d'Apollon Dionysodotes 51 . Cette épithète se rapporte à une variante du mythe de Zagreus $^{52}$, selon laquelle Apollon, après le démembrement de Dionysos par les Titans, l'avait ressuscité et offert aux mortels en signe de réconciliation entre l'esprit apollinien et l'esprit bacchique. Et cette réconciliation, qui constitue le principe par excellence de l'orphisme, s'illustre au mieux dans les œuvres littéraires et artistiques qui ont trait au grand sanctuaire delphique que les deux dieux se partageaient l'année durant ${ }^{53}$.

Institut de Recherches Humanistes

Ioannis LOUCAS

Acadimias, 91-93

GR - 10677 ATHÈNES

50 BOEHME, op. cit., p. 314-317.

51 Paus., I, 31, 4 : étant donné que le texte du Périégète comporte la mention de ce

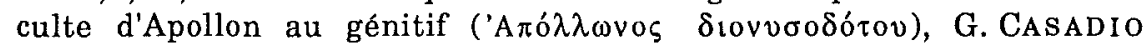
(Antropologia gnostica e antropologia orfica nelle notizia di Ippolito sui Sethiani, in Sangue e antropologia nelle teologia, Rome, à paraître en novembre 1989 , p. $42-43$ du tiré à part) propose que l'on restitue le nominatif

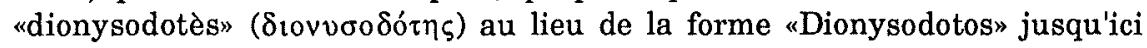
couramment admise.

52 OLYMPIODORE, fr. 209 Kern, p. 227. Cf. les remarques de N. PAPACHATZIS dans son c.r. de mon ouvrage Rhéa-Cybèle, in Kernos, 2 (1989), p. 267-268.

53 Cf., entre autres, Delcourt, op. cit., p. 194-200; H. Jeanmaire, Dionysos. Histoire du culte de Bacchus, Paris, 1951 [1978], p. 194-196; Roux, op. cit., p. 175-184. 